\title{
Effect of cricoid pressure on laryngeal view during prehospital tracheal intubation: a propensity-based analysis
}

\author{
Emmanuel Caruana, ${ }^{1,2}$ Sylvie Chevret, ${ }^{1}$ Romain Pirracchio ${ }^{2,3,4}$
}

'Service Mobile d'Urgences et Réanimation, Hôpital Beaujon, Clichy, France

${ }^{2}$ Service de Biostatistiques et Informatique Médicale, Hôpital Saint-Louis, AP-HP; INSERM 1153 ECSTRA Team; Université Paris 7 Diderot, Paris, France ${ }^{3}$ Service de Biostatistique et Information Médicale, Hôpital Européen Georges Pompidou, Université Paris V DescartesSorbonne Paris Cité, Paris, France

${ }^{4}$ Department of Anesthesia and Perioperative Care, San Francisco General Hospital \& Trauma Center, University of California San Francisco, San Francisco, California, USA

\section{Correspondence to}

Dr Emmanuel Caruana, A\&E Department, Beaujon hospital university AP-HP, 100, boulevard du Général Leclerc, Clichy 92110, France:

manucaru@hotmail.com

Received 15 January 2016 Revised 11 September 2016 Accepted 12 September 2016 Published Online First 30 September 2016

\section{ABSTRACT}

Background The benefit of cricoid pressure during tracheal intubation is still debated and, due to its potential negative impact on laryngeal views, its routine use is questioned. The goal of this study was to estimate its impact on laryngeal view.

Methods All patients intubated in the prehospital setting were included. Three different propensity score (PS) models were used and compared in terms of the balance achieved between those patients who received cricoid pressure and those who did not. The PS model that optimised the balance was retained in order to estimate the relationship between cricoid pressure and the following outcomes: difficult laryngoscopy, intubation-related complications and difficult intubation. Results Among the 1195 patients included, 499 $(41.7 \%)$ received cricoid pressure. The optimal PS included seven variables (cardiac arrest, altered neurological status, shock, respiratory distress, gender, obesity, patient's position). After PS matching, no significant risk difference (RD) in the rate of difficult laryngoscopy was found between the patients who received cricoid pressure and those who did not $(R D=0.001,95 \% \mathrm{Cl}-0.07$ to $0.08, \mathrm{p}=0.50$ ). No significant difference was found in terms of difficult intubation ( $R D=0.06,95 \% \mathrm{Cl}-0.13$ to $0.25, \mathrm{p}=0.28$ ) and in terms of prevalence of complications, except for airway trauma that were more frequent in cricoid pressure group ( $R D=0.03,95 \% \mathrm{Cl} 0.002$ to $0.05, p=0.01$ ).

Conclusions No significant relationship was found between the use of cricoid pressure for prehospital intubation and difficult laryngoscopy. Cricoid pressure was found to be associated with more airway trauma. This finding could question its routine use.

\section{INTRODUCTION}

Out-of-hospital airway management can be particularly cumbersome, with poor tracheal intubation conditions. Cricoid pressure, also referred to as the Sellick manoeuvre, was described in 1961 to prevent regurgitation of gastric content during anaesthesia induction. ${ }^{1}$ This manoeuvre aims at transiently occluding the upper end of the oesophagus through the application of a backward pressure on cricoid cartilage against the cervical spine. Such cricoid pressure results in the compression of the postcricoid hypopharynx and should prevent gastric regurgitation into the pharynx. Guidelines for cardiopulmonary resuscitation and emergency cardiovascular care recommend the use of cricoid pressure for intubation in all patients, except those in cardiac arrest, ${ }^{2}$ and especially in trauma patients.

\section{Key messages}

What is already known on this subject?

- Cricoid pressure is widely used to reduce the incidence of gastric content aspiration during anaesthesia induction, but its potential adverse impact on laryngeal views challenged its routine use.

\section{What this study adds?}

- Cricoid pressure is widely used to reduce the incidence of gastric content aspiration during intubation, but previous literature has suggested it may have a negative impact on laryngeal views.

- In this study of 1195 patients intubated in the prehospital setting, we used a propensity score to account for potential selection bias in the use of cricoid pressure. Cricoid pressure had no impact on laryngeal views and did not increase the rate of difficult intubations. Cricoid pressure was associated with more airway trauma.

However, the expected benefit and thus the need to use cricoid pressure are still debated in the literature. First, there is clinical evidence that cricoid pressure might fail to prevent aspiration during intubation. ${ }^{3}$ In addition, several studies have reported a negative impact on airway patency and therefore on ventilation. ${ }^{4}$

The results concerning the impact of cricoid pressure on laryngeal view are conflicting and come either from cadaver studies or from small studies including patients intubated in the operating room for elective surgery. Hence, there is a lack of robust information on the impact of cricoid pressure on laryngoscopic conditions during urgent intubation. In this context, any credible proof of a link between the use of cricoid pressure and an increase rate of difficult laryngoscopy would be a strong argument against its routine use.

Causal inference methods have been suggested in order to estimate causal relationships between an intervention and an outcome, when the study is potentially confounded by selection bias due to the absence of randomisation. ${ }^{5}$ Among other things, propensity score (PS) matching has been shown to well balance measured baseline covariates across treatment groups and thus to better estimate the causal relationship between the exposure and the outcome. 
The primary goal of this study was to use a propensity-based approach to estimate the impact of cricoid pressure on the rate of difficult laryngoscopy based on a prospective observational study of patients intubated outside the hospital.

\section{METHODS}

\section{Study design}

All consecutive patients intubated in the prehospital setting between April 2008 and November 2012 by a French physicianstaffed emergency medical services (EMS) unit from a teaching hospital were prospectively included in a registry. The exclusion criteria were anyone aged under 18, contraindications to succinylcholine (ie, known allergy, malignant hyperthermia, myopathy, tetraplegia, preeclampsia and hyperkalemia), ketamine or etomidate. The study was based on this registry, which was declared to the French data protection authority (Commission Nationale de l'Informatique et des Libertés, number of declaration $1674124 \mathrm{v} 0)$.

\section{Setting}

Our EMS team is composed of at least one ambulance driver, an anaesthetic nurse and a senior emergency physician. Residents in their last year of training may also be present. All emergency physicians are board-certified in emergency medicine, that is, they have at least a 2-year experience in emergency medicine in addition to their 3-year residency. They all received theoretical and practical training in emergency airway management. All residents have to attend an initial training for intubation in the operating theatre. To be completed, this training requires at least 10 successive successful intubations.

The team followed a standardised intubation procedure: the patient's position was optimised for tracheal intubation (TI) by placing the patients on a stretcher at full height before intubation. After a 3-min preoxygenation period, all patients received a rapid sequence intubation with either etomidate $(0.3 \mathrm{mg} / \mathrm{kg})$, ketamine $(2 \mathrm{mg} / \mathrm{kg})$ or thiopentone $(3-5 \mathrm{mg} / \mathrm{kg})$ in association with succinylcholine $(1-1.5 \mathrm{mg} / \mathrm{kg})$, based on the guidelines from the French Society of Emergency Medicine. The physician in charge decided whether cricoid pressure should be applied or not. Cricoid pressure was performed by a nurse anaesthetist with substantial experience in both EMS and operating theatres, and using the method described by Sellick. ${ }^{1}$ This procedure involved careful identification of cricoid cartilage with the thumb and index finger, followed by the application of a steady pressure mainly by the index finger. A force of $30 \mathrm{~N}$ was considered desirable $^{6}$ but could not be measured. Patients in cardiac arrest were intubated without preoxygenation or induction, their position was optimised on a stretcher at full height whenever possible. Either single-use or reusable laryngoscope blades (Macintosh size 4) were used. The intubation algorithm was based on the guidelines endorsed by the French Society of Anesthesiology and Intensive Care Medicine. ${ }^{7}$ After a first attempt, or in case of a suspected difficult airway, the physicians were encouraged to follow a two-step predefined airway management algorithm: first, direct laryngoscopy with an external laryngeal manipulation and/or the use of alternative techniques such as Eschmann bougie; second, in case of failure, tracheal intubation through a laryngeal mask. Waveform capnography was used in all patients.

\section{Data collection}

The physicians collected clinical data immediately after the intervention. One of the physicians from the EMS unit was responsible for quality assessment throughout the study. The baseline variables were age, gender, obesity (0: absence, 1: presence) defined by a body mass index over 30 (estimated from the height and weight measures reported by the patient or his/ her next of kin when available, or estimated by the physician when unavailable), reasons for intubation (cardiac arrest, altered neurological status, ie, coma/agitation/drug intoxication/trauma, acute respiratory distress and shock), patient's position during the procedure (on the floor or on a stretcher at full height), operator's status (senior or resident) and presence of cervical immobilisation.

\section{Outcomes}

The primary outcome was the rate of difficult laryngoscopy as defined by the Cormack and Lehane ${ }^{8}(\mathrm{CL})$. The CL score classifies laryngoscopic views in four grades (I, full view of glottis; II, partial view of glottis; III, only epiglottis seen, glottis not seen; IV, neither glottis nor epiglottis seen). The physician who performed the intubation reported the CL grade. A difficult laryngoscopy was defined as a CL grade III or IV. ${ }^{9}$

The secondary outcomes were the rate of difficult intubation as defined by two or more failed laryngoscopic attempts or the need for any alternative methods for intubation (as defined by the French Society of Anesthesiology and Intensive Care Medicine $)^{7}$; and the number of intubation-related complications occurring within $5 \mathrm{~min}$ after the procedure: oxygen desaturation $\left(\mathrm{SpO}_{2}<90 \%\right)$, aspiration (regurgitation visualised during the laryngoscopy), vomiting, bronchospasm and/or laryngospasm, mainstem intubation, recognised or unrecognised oesophageal intubation (diagnosed using a waveform capnography/capnometry at sixth breath combined with pulmonary auscultation) and airway trauma.

\section{Statistical analysis}

Continuous variables are expressed as mean with SD or median with IQR when appropriate. Counts and percentages are provided for categorical variables.

We used a PS approach to account for selection bias. ${ }^{5}$ The PS was defined as the individual probability of receiving cricoid pressure given baseline observed covariates. This probability was estimated using a logistic regression model with cricoid pressure as dependent variable. Several PS models were tested, each characterised by a different set of explanatory variables.

- The first PS model (PS 1) included all collected baseline variables.

- The second PS model (PS 2) included (i) confounders: variables suspected to be related to both the treatment and the outcome and (ii) prognostic factors: strong predictors of the outcome but not of treatment allocation. A third type of variables, called instrumental variables, are variables that would act as strong predictors of treatment allocation but not of the outcome; however, instrumental variables in the PS model may jeopardise the efficiency of the estimator and thus were not included. ${ }^{10}$ Based on subject-matter knowledge and significant statistical association in multivariate analysis, we identified four confounders (cardiac arrest, altered neurological status, shock and respiratory distress) and three prognostic factors (gender, obesity and patient's position). All seven variables were included in the PS 2.

- For the third PS model (PS 3), we only included statistically significant prognostic variables, as identified using a multivariate logistic regression model with a stepwise selection procedure based on the Akaike Information Criterion. Three variables were selected and included into the PS model 3: gender, patient's position and obesity. 
The three different PS models were then used to match the patients who received cricoid pressure to those who did not receive it. The nearest neighbour matching algorithm allowed for replacement and for ties, so that subjects not exposed to cricoid pressure could be matched to several subjects with cricoid pressure if their PS were equal or close.

The balance in the distribution of baseline covariates was checked using the standardised mean difference (SMD). A SMD greater than $0.1(10 \%)$ denotes meaningful imbalance in baseline covariates. ${ }^{10}$ Caruana et al ${ }^{11}$ recently proposed a weighted balance measure (WBM) that takes into account the strength of association between each covariate and the outcome. We defined the best PS model as the one optimising covariate balance and thereby minimising the WBM.

The average treatment effect on the treated (expressed as a risk difference together with its 95\% CI) was defined as the parameter of interest. Variance estimation was based on the Abadie-Imbens robust variance estimator. Fifty-six (4\%) patients with missing data were excluded from the analysis. Missing values mainly concerned the variable 'obesity', which was not available in 43 patients $(3 \%, 27$ non-exposed patients and 16 exposed patients).

All statistical tests were two-tailed, and $\mathrm{p}<0.05$ considered statistically significant. Bonferroni correction (ie, $p$ values multiplied by the number of comparisons, ie, eight) was used to handle the risk of type I error inflation due to multiple comparisons in the univariate analyses. Due to the use of matching with replacement, secondary outcomes were compared in PS matched sample with the risk difference and its 95\% CI.

All analyses were performed using R.3.2.0 statistical software on a Mac OsX platform.

\section{RESULTS}

\section{Study population}

During the study period, 1291 patients were intubated. Thirty-two patients aged more than 18 years, 8 adults with a contraindication to succinylcholine (rocuronium used instead), and 56 patients with missing data were excluded from the final analysis that included a total of 1195 patients (figure 1). Baseline characteristics are reported in table 1. In the original sample, the main observed differences between groups concerned the following variables: altered neurological status $(59 \%$ in the cricoid pressure group vs $8 \%$ in the non-cricoid pressure group, $\mathrm{SMD}=1.05)$, patient's position $(56 \%$ in the cricoid pressure group vs $12 \%, \mathrm{SMD}=0.88)$ and age (mean $=63(\mathrm{SD}=18)$ in the cricoid pressure group vs mean $=57(\mathrm{SD}=18), \mathrm{SMD}=$ -0.33). As expected, patients intubated for cardiac arrest were more prevalent in the non-cricoid pressure group ( $83 \%$ vs $18 \%$, $\mathrm{SMD}=-1.75$ ).

\section{Propensity score matching and balance measures}

The SMDs for the original and the matched datasets are reported in table 1 . The best overall balance was obtained for PS 2 that included the confounders and the prognostic factors (figure 2). Accordingly, the WBM was minimised by the PS 2 (WBM=0.41). Therefore, the second PS model (PS 2) was selected as the best PS model for further analyses. Age was the only variable that remained slightly imbalanced after matching $(\mathrm{SMD}=-0.15$ for the PS 2).

\section{Outcomes}

Estimated effects of cricoid pressure in the original cohort and in PS 2 matched cohort are reported in table 2. In the original cohort (risk difference $=-0.03,95 \%$ CI -0.17 to $0.18, p=0.37$ ) as well as in the matched cohort (risk difference $=0.001,95 \%$ CI -0.07 to $0.08, p=0.50$ ), the rate of difficult laryngoscopy did not differ between treatment groups. Further adjustment on age to account for residual imbalance did not markedly alter the results (risk difference $=0.01,95 \% \mathrm{CI}-0.06$ to $0.08, \mathrm{p}=0.39$ ).

The risk of difficult intubation was similar in the cricoid pressure and the non-cricoid pressure group in the original sample (risk difference $=-0.02,95 \% \mathrm{CI}-0.22$ to $0.17, \mathrm{p}=0.43$ ), as

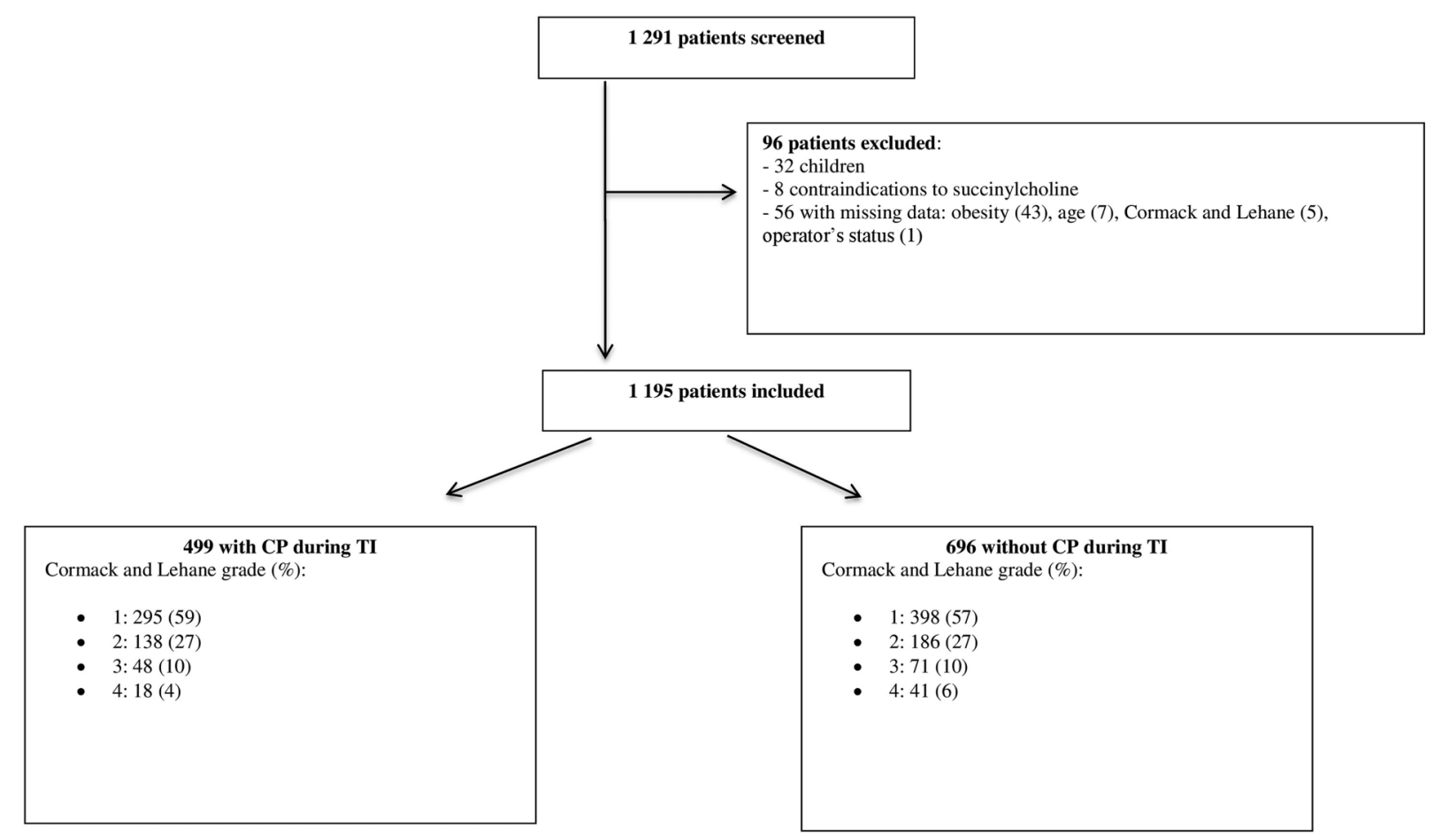

Figure 1 Study flow chart 
Table 1 Baseline characteristics balance in the original ant matched cohort for each PS models

\begin{tabular}{|c|c|c|c|c|c|c|}
\hline \multirow[b]{2}{*}{ Characteristics } & \multicolumn{3}{|c|}{ Overall cohort $(n=1195)$} & \multirow{2}{*}{$\begin{array}{l}\text { PS } 1 \text { matched cohort } \\
\text { SMD* }\end{array}$} & \multirow{2}{*}{$\begin{array}{l}\text { PS } 2 \text { matched cohort } \\
\text { SMD* }\end{array}$} & \multirow{2}{*}{$\begin{array}{l}\text { PS } 3 \text { matched cohort } \\
\text { SMD* }\end{array}$} \\
\hline & $\begin{array}{l}\text { No cricoid pressure } \\
(n=696)\end{array}$ & $\begin{array}{l}\text { Cricoid pressure } \\
(n=499)\end{array}$ & SMD* & & & \\
\hline Age (mean, SD) & $63(18)$ & $57(18)$ & -0.33 & $-0.02 \dagger$ & -0.15 & -0.22 \\
\hline Gender (female) (n, \%) & $245(35)$ & $190(38)$ & -0.06 & $0.09+$ & 0 & $0 \dagger$ \\
\hline Obesity (n, \%) & 97 (14) & $57(11)$ & -0.08 & $-0.03 \dagger$ & $0.03 t$ & $0+$ \\
\hline Operator's status (senior) (n, \%) & $318(46)$ & $211(42)$ & -0.07 & $0.04 t$ & $-0.07 \dagger$ & -0.09 \\
\hline Patient's position $\ddagger(n, \%)$ & $87(12)$ & $280(56)$ & 0.88 & $0+$ & $0+$ & $0+$ \\
\hline Cervical immobilisation (n, \%) & $55(8)$ & $51(10)$ & 0.08 & $-0.04 \dagger$ & -0.02 & -0.10 \\
\hline \multicolumn{7}{|l|}{ Reasons for intubation (n, \%) } \\
\hline Cardiac arrest & $576(83)$ & $89(18)$ & -1.75 & $0 \dagger$ & $0 \dagger$ & -0.90 \\
\hline Altered neurological status & $53(8)$ & $295(59)$ & 1.05 & $-0.05 t$ & $0 \dagger$ & 0.73 \\
\hline Acute respiratory distress & $22(3)$ & $32(6)$ & 0.13 & $-0.02 \dagger$ & $-0.02 \dagger$ & -0.15 \\
\hline \multirow[t]{4}{*}{ Shock } & $29(4)$ & $83(17)$ & 0.33 & $0.09+$ & $0.01 \dagger$ & 0.05 \\
\hline & \multicolumn{6}{|c|}{ Weighted balance measure } \\
\hline & \multicolumn{2}{|c|}{ Overall cohort $(n=1195)$} & & PS 1 matched cohort & PS 2 matched cohort & PS 3 matched cohort \\
\hline & \multicolumn{2}{|l|}{29.70} & & 1.48 & 0.41 & 15.58 \\
\hline \multicolumn{7}{|c|}{$\begin{array}{l}\text { *SMD is the mean difference divided by the pooled SD. } \\
\text { †Variables included in each PS model. } \\
\text { ¥Stretcher at full height. } \\
\text { PS, propensity score; SMD, standardised mean difference. }\end{array}$} \\
\hline
\end{tabular}

Figure 2 Standardised mean differences before and after matching. PS, propensity score.

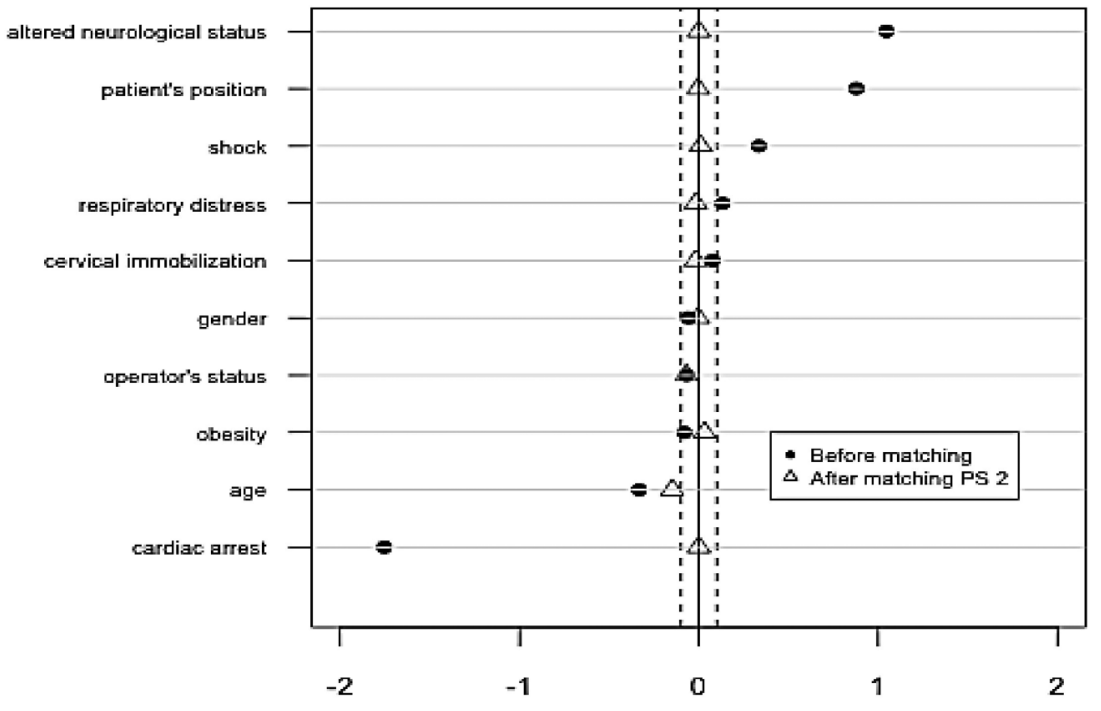

Standardized difference well as in the matched sample (risk difference $=0.06,95 \% \mathrm{CI}$ -0.13 to $0.25, \mathrm{p}=0.28$ ).

A total of 195 intubation-related complications occurred in 153 patients (13\%). In the original cohort (before matching), more intubation-related complications were observed in cricoid pressure group $(n=84(17 \%)$ vs $69(10 \%), p<0.001$; risk difference $=0.07,95 \%$ CI -0.05 to $0.19, \mathrm{p}=0.13)$. Oesophageal intubation $(n=29,6 \%)$ and desaturation $(n=40,8 \%)$ were the most frequent complications. When patients were exposed to cricoid pressure, oesophageal intubation occurred less frequently with a stretcher at full height $(38 \%$ vs $62 \%, \mathrm{p}=0.04$, data not shown). After matching, there was no difference in terms of complications between patients exposed to cricoid pressure and those unexposed to it, except for airway trauma that were more frequent in cricoid pressure group with $3.6 \%$ as compared with $0.5 \%$ in non-cricoid pressure group (risk difference $=0.03,95 \%$ CI 0.002 to $0.05, \mathrm{p}=0.01$ ).

\section{DISCUSSION}

Despite the lack of scientific evidence, cricoid pressure is widely used during anaesthesia induction to reduce the incidence of gastric content aspiration. In emergency medicine, the efficiency of cricoid pressure to prevent aspiration still has to be documented. ${ }^{12}$ In addition, the use of cricoid pressure during emergent intubation might be associated with impaired laryngeal views. ${ }^{13}$ The primary objective of this study was to assess the impact of cricoid pressure on laryngeal view based on a large prehospital observational dataset. Using a PS matching approach, no 
Table 2 Difficult laryngoscopy, complication and difficult intubation according to cricoid pressure during tracheal intubation

\begin{tabular}{|c|c|c|c|c|}
\hline \multirow[b]{2}{*}{ Outcome } & \multicolumn{2}{|l|}{ Overall cohort } & \multirow[b]{2}{*}{ p Value* } & \multirow{2}{*}{$\begin{array}{l}\text { PS } 2 \text { matched cohort } \\
\text { Risk difference }(95 \% \mathrm{CI})\end{array}$} \\
\hline & No cricoid pressure $(n=696)$ & Cricoid pressure $(n=499)$ & & \\
\hline Difficult laryngoscopy (n, \%) & $112(17)$ & $66(13)$ & & \\
\hline Risk difference $(95 \% \mathrm{Cl})$ & $-0.03(-0.07$ to 0.01$)$ & & & $0.001(-0.07$ to 0.08$)$ \\
\hline Complication (n, \%) & $69(10)$ & $84(17)$ & $<0.001$ & $0.04(-0.06$ to 0.15$)$ \\
\hline Oesophageal intubation & $34(5)$ & $29(6)$ & 0.47 & $0.05(-0.03$ to 0.13$)$ \\
\hline Desaturation & $14(2)$ & $40(8)$ & $<0.001$ & $-0.005(-0.05,0.04)$ \\
\hline Mainstem intubation & $16(2)$ & $18(4)$ & 0.17 & $0.016(-0.02$ to 0.05$)$ \\
\hline Trauma & $4(1)$ & $11(2)$ & 0.01 & $0.03(0.002$ to 0.05$)$ \\
\hline Vomiting, aspiration during intubation & $5(1)$ & $14(3)$ & 0.004 & $0.02(-0.006$ to 0.05$)$ \\
\hline Bronchospasm, laryngospasm & $6(1)$ & $1(0)$ & 0.27 & $-0.005(-0.03$ to 0.02$)$ \\
\hline Difficult intubation (n, \%) & $283(41)$ & $190(38)$ & 0.36 & $0.06(-0.13$ to 0.25$)$ \\
\hline
\end{tabular}

significant relationship was found between the use of cricoid pressure and the rate of difficult laryngoscopy as defined by a $\mathrm{CL}^{8}$ grade III or IV. This result is consistent with a previous randomised controlled study ${ }^{14}$ reporting no association between the CL grade and the use of cricoid pressure in the operating theatre.

In the original sample (before matching), intubation-related complications were significantly less frequent in the group of patients without cricoid pressure. As a potential explanation, this group encompassed more cardiac arrests, known to be associated with better intubation conditions. However, oesophageal intubation tended to be more frequent in cardiac arrest patients unexposed to cricoid pressure. Suboptimal patient's position may in turn explain this result. Indeed optimal patient positioning is known to be associated with better intubation conditions. In the matched sample however, airway trauma were more frequent in the cricoid pressure group. No significant risk difference was observed in terms of pulmonary aspirations. However, given its low incidence, this study was clearly not powerful enough to conclude on the effect of cricoid pressure on preventing aspiration episodes.

For the patients in cardiac arrest, the use of cricoid pressure is not clearly recommended in the guidelines and is therefore less commonly used. Accordingly, in our sample, most of the cardiac arrests were observed in the non-exposed group and it was often difficult to match them with subject who received cricoid pressure. In this setting where there are few control individuals comparable to the treated individuals, it is helpful to use matching with replacement because controls that look similar to many treated individuals can be used multiple times. ${ }^{15}$ In this situation, nearest neighbour matching may result in bad matches. However, this risk was limited by allowing replacement, which in turn increases the overall balance and may in turn decrease the bias. ${ }^{16}$ Furthermore, this situation constitutes a threat for the positivity assumption. ${ }^{17}$ Positivity is one of the key assumptions in causal inference and basically requires both exposed and unexposed observations to be represented for each possible covariate combination. Here, because almost all patients in cardiac arrest did not receive cricoid pressure, this assumption can be considered as nearly violated. The most appropriate way to interpret this result is to consider that the conclusions only apply to the subjects they were actually matched to. ${ }^{18}$ Hence our results should not be extrapolated to patients intubated for a cardiac arrest.
The goal of PS matching is to create a counterfactual group where observed relevant baseline patient characteristics are balanced across groups. ${ }^{19}$ PS matching may sometimes miss its target, especially when baseline imbalance is limited. In this case, PS matching may result in discarding part of the population and thus may decrease the efficiency of the estimator. As highlighted by King and Nielsen in their working paper, ${ }^{20}$ the first step is to clearly define the causal quantity of interest. The average treatment effect in the treated, which may be more straightforwardly targeted by PS matching estimators, was the quantity of interest in our study. ${ }^{21}$ A second point is to verify that the distribution of baseline covariates in the treated and the controls is indeed imbalance. This was indeed the case in the present sample. Finally, PS model specification is of paramount importance both in terms of the variables included in the model $^{11}$ and the functional form of the relationship between treatment allocation and the explanatory variables. ${ }^{22}$ After matching, the best PS model is then the one that offers the best balance across groups. Multiple balance metrics have been proposed in this context, the most widely used in practice being the average SMD, that is, the empirical mean of the standardised difference obtained for each single covariate. ${ }^{23}$ The major drawback of this metric is that it attributes the same weight to each patient characteristic, while, in practice, it may be more important to balance one variable than another. For instance, in the present case, it seems more important to balance the distribution of patients with obesity than the distribution of gender. An alternative metric, referred to as the $\mathrm{WBM}^{11}{ }^{11}$ has been recently developed to take into account the strength of association between the covariates and the outcome. The goal is to emphasise the need to adequately balance the distribution of the covariates strongly associated with the outcome. Explanatory variables were classified into confounders, prognostic factors and instrumental variables based on expert-matter knowledge and on a quantification of their association with the outcome using multivariable logistic models. Subsequently, three PS models were tested with the goal to only include in the PS model the minimal set of covariates to minimise the WBM. Although PS matching succeeded in reducing the WBM and balancing most patient characteristics at baseline, patient's age was not evenly distributed after matching. Thus, the effect of cricoid pressure on the outcome was also estimated, in the matched dataset, using a regression model adjusting for age. Adjusting on 
age did not modify the estimated association between cricoid pressure and the outcome. Patients in cardiac arrest were older and usually unexposed to cricoid pressure; however, adjusting on age did not change this final result.

This study carries some limitations. First, no measure of the actual pressure produced by cricoid pressure was possible in prehospital conditions. However, highly trained personnel only performed cricoid pressure and tracheal intubation. Second, the CL grade relies on a subjective assessment of the vocal chords visualisation. Such a subjective rating might have introduced a certain degree of evaluation bias. This classification is indeed known to carry a limited interobserver reliability and a poor intraobserver reproducibility, both questioning the validity of the CL classification to document laryngeal view during direct laryngoscopy. ${ }^{24}$ Moreover, no subjective assessment of the laryngeal view was collected before its application to detect if cricoid pressure could enhance the laryngeal view for patients with CL grade IV. Further works are needed to detail this point. Third, the impact of backward upward rightward pressure could not be specifically addressed in this study as the CL grade was collected before any external manoeuvre. Finally, as previously stated, our conclusions only apply to a population where it was indeed possible to compare exposure to unexposure to cricoid pressure. Therefore, further studies would be needed to answer the question of the benefit of cricoid pressure in patients intubated for cardiac arrest.

In summary, cricoid pressure used for prehospital emergent tracheal intubation is not associated with difficult laryngoscopy as defined by the CL grade III or IV. However, in our cohort, its use was associated with more airway trauma. In this context, the real benefit of cricoid pressure to prevent gastric content aspiration still has to be demonstrated.

Acknowledgements François Xavier Duchateau, who provided the dataset.

Contributors EC performed the analysis and wrote the manuscript. SC supervised the study and helped draft the manuscript. RP contributed to the statistical analysis and helped draft the manuscript. All authors read and approved the final manuscript.

Competing interests None declared.

Ethics approval Commission Nationale de I'Informatique et des Libertés.

Provenance and peer review Not commissioned; externally peer reviewed.

\section{REFERENCES}

1 Sellick BA. Cricoid pressure to control regurgitation of stomach contents during induction of anaesthesia. Lancet 1961:2:404-6.
2 Link MS, Berkow LC, Kudenchuk PJ, et al. Part 7: adult advanced cardiovascular life support 2015 American Heart Association guidelines update for cardiopulmonary resuscitation and emergency cardiovascular care. Circulation 2015;132:S444-64.

3 Schwartz DE, Matthay MA, Cohen NH. Death and other complications of emergency airway management in critically ill adults. A prospective investigation of 297 tracheal intubations. Anesthesiology 1995;82:367-76.

4 Hartsilver EL, Vanner RG. Airway obstruction with cricoid pressure. Anaesthesia 2000:55:208-11.

5 Rosenbaum PR, Rubin DB. The central role of the propensity score in observational studies for causal effects. Biometrika 1983;70:41-55.

6 Vanner RG, Asai T. Safe use of cricoid pressure. Anaesthesia 1999;54:1-3.

7 Société Française d'Anesthésie et de Réanimation. Intubation difficile. 2006.

8 Cormack RS, Lehane J. Difficult tracheal intubation in obstetrics. Anaesthesia 1984;39:1105-11.

9 Langeron 0, Birenbaum A, Le Saché F, et al. Airway management in obese patient. Minerva Anestesiol 2014;80:382-92.

10 Austin PC. Balance diagnostics for comparing the distribution of baseline covariates between treatment groups in propensity-score matched samples. Stat Med 2009:28:3083-107.

11 Caruana E, Chevret S, Resche-Rigon M, et al. A new weighted balance measure helped to select the variables to be included in a propensity score model. I Clin Epidemiol 2015;68:1415-22.e2.

12 Trethewy $C E$, Burrows JM, Clausen $D$, et al. Effectiveness of cricoid pressure in preventing gastric aspiration during rapid sequence intubation in the emergency department: study protocol for a randomised controlled trial. Trials 2012;13:17.

13 Ellis DY, Harris T, Zideman D. Cricoid pressure in emergency department rapid sequence tracheal intubations: a risk-benefit analysis. Annal Emerg Med 2007:50:653-65

14 Turgeon AF, Nicole PC, Trépanier CA, et al. Cricoid pressure does not increase the rate of failed intubation by direct laryngoscopy in adults. Anesthesiology 2005;102:315-19.

15 Dehejia RH, Wahba $\mathrm{S}$. Causal effects in nonexperimental studies: reevaluating the evaluation of training programs. J Am Stat Assoc 1999;94:1053-62.

16 Caliendo M, Kopeinig S. Some practical guidance for the implementation of propensity score matching. J Econ Surveys 2008;22:31-72.

17 Westreich D, Cole SR. Invited commentary: positivity in practice. Am I Epidemiol 2010:171:674-7: discussion 678-81.

18 Petersen ML, Porter KE, Gruber S, et al. Diagnosing and responding to violations in the positivity assumption. Stat Methods Med Res 2012:21:31-54.

19 Stuart EA. Matching methods for causal inference: a review and a look forward. Stat Sci 2010;25:1-21.

20 King G, Nielsen R. Why propensity scores should not be used for matching. Copy at http://jmp/1sexgVw Export BibTex Tagged XML Download Paper 2015;481.

21 Pirracchio R, Carone M, Rigon MR, et al. Propensity score estimators for the average treatment effect and the average treatment effect on the treated may yield very different estimates. Stat Methods Med Res 2013. Published Online First: 6 November 2013

22 Pirracchio R, Petersen ML, van der Laan M. Improving propensity score estimators robustness to model misspecification using super learner. Am J Epidemiol 2015:181:108-19.

23 Austin PC. Assessing balance in measured baseline covariates when using many-to-one matching on the propensity-score. Pharmacoepidem Drug Safe 2008:17:1218-25.

24 Krage R, van Rijn C, van Groeningen D, et al. Cormack-Lehane classification revisited. Br J Anaesth 2010:105:220-7. 